September 9, 2003

revised on October 7, 2003

hep-th/0309091

\title{
Quantization on a torus without position operators $^{1}$
}

\author{
Shogo Tanimura ${ }^{2}$ \\ Graduate School of Engineering, Osaka City University \\ Sugimoto, Sumiyoshi-ku, Osaka 558-8585, Japan
}

\begin{abstract}
We formulate quantum mechanics in the two-dimensional torus without using position operators. We define an algebra with only momentum operators and shift operators and construct an irreducible representation of the algebra. We show that it realizes quantum mechanics of a charged particle in a uniform magnetic field. We prove that any irreducible representation of the algebra is unitarily equivalent to each other. This work provides a firm foundation for the noncommutative torus theory.
\end{abstract}

PACS: 02.40.Gh; 03.65.Ca; 03.65.Fd; 04.60.Ds

\footnotetext{
${ }^{1}$ To be published in Modern Physics Letters A.

${ }^{2}$ e-mail: tanimura@mech.eng.osaka-cu.ac.jp
} 


\section{Introduction}

Traditionally quantum mechanics in the Euclidean space $\boldsymbol{R}^{n}$ is defined as an irreducible representation of the canonical commutation relation (CCR). Namely, self-adjoint operators $\hat{q}_{j}, \hat{p}_{j}(j=1,2, \cdots, n)$ are required to satisfy the CCR

$$
\left[\hat{q}_{j}, \hat{p}_{k}\right]=i \delta_{j k} \hat{1}, \quad\left[\hat{q}_{j}, \hat{q}_{k}\right]=\left[\hat{p}_{j}, \hat{p}_{k}\right]=0
$$

and the algebra generated by $\left\{\hat{q}_{j}, \hat{p}_{j}\right\}$ is represented on the Hilbert space $L^{2}\left(\boldsymbol{R}^{n}\right)=\{\psi$ : $\left.\boldsymbol{R}^{n} \rightarrow \boldsymbol{C}\right\}$, which consists of square integrable functions, as

$$
\hat{q}_{j} \psi(q)=q_{j} \psi(q), \quad \hat{p}_{j} \psi(q)=-i \frac{\partial}{\partial q_{j}} \psi(q) .
$$

This is called the Schrödinger representation. The operator $\hat{q}_{j}$ is called the position operator while $\hat{p}_{j}$ is called the momentum operator. It has been proved[1] that any other irreducible representation of the CCR is unitarily equivalent to the Schrödinger representation although there still remains subtlety in the argument [2, 3].

When we formulate quantum mechanics in the circle $S^{1}$, we need to introduce an algebra that is different from the CCR. A wave function in $S^{1}$ is demanded to be a periodic function such that $\psi(q+2 \pi)=\psi(q)$. However, when it is multiplied by $q, q \psi(q)$ is not a periodic function. Hence the position operator $\hat{q}$ is not well defined in $S^{1}$. An alternative formulation of quantum mechanics in $S^{1}$ has been given [4]- [7].

When we formulate quantum mechanics in $\boldsymbol{R}^{2}$ with a uniform background magnetic field $B$, we have no trouble. We can write down the Hamiltonian

$$
\hat{H}=\frac{1}{2}\left(\hat{p}_{1}\right)^{2}+\frac{1}{2}\left(\hat{p}_{2}-B \hat{q}_{1}\right)^{2}
$$

which describe dynamics of a charged particle in the magnetic field.

When we formulate quantum mechanics in the torus $T^{2}$ with the background magnetic field, we have trouble. The torus has a coordinate system $\left(q_{1}, q_{2}\right) \in \boldsymbol{R}^{2}$. For arbitrary integers $\left(n_{1}, n_{2}\right)$, the point $\left(q_{1}+2 \pi n_{1}, q_{2}+2 \pi n_{2}\right)$ is to be identified with $\left(q_{1}, q_{2}\right)$. Thus, the operator $\hat{q}_{1}$ that appears in (1.3) is not well defined and, therefore, the Hamiltonian (1.3) is not well defined, either. So we need to find another formulation of quantum mechanics in $T^{2}$ with the magnetic field.

In this Letter we solve this problem. We define an algebra $\mathcal{A}$ without using position operators, instead using shift operators that characterize symmetry of the torus. We then show that an irreducible representation of the algebra $\mathcal{A}$ realizes quantum mechanics in the torus with the uniform magnetic field. Moreover, we introduce other operators which can be interpreted as a kind of position operators, and define another algebra $\mathcal{A}^{\prime}$ using them. We show that $\mathcal{A}^{\prime}$ is actually equivalent to $\mathcal{A}$. So, quantization without position operators is possible and it reproduces position operators, too. 


\section{Main results}

Here we describe definitions and main theorems concisely. In the rest of Letter we will prove them.

Definition 1: quantization without position operators. Let $m$ be an integer. Assume that there are self-adjoint operators $\hat{P}_{j}$ and unitary operators $\hat{V}_{j}(j=1,2)$ which satisfy the following relations

$$
\begin{aligned}
& {\left[\hat{P}_{1}, \hat{P}_{2}\right]=\frac{i m}{2 \pi} \hat{1},} \\
& \left(\hat{V}_{1}\right)^{m}=\left(\hat{V}_{2}\right)^{m}=\hat{1}, \\
& \hat{V}_{1} \hat{V}_{2}=e^{2 \pi i / m} \hat{V}_{2} \hat{V}_{1}, \\
& {\left[\hat{P}_{j}, \hat{V}_{k}\right]=0 .}
\end{aligned}
$$

The operators $\hat{P}_{j}$ are called momentum operators while the operators $\hat{V}_{j}$ are called shift operators. The algebra $\mathcal{A}$ generated by $\left\{\hat{P}_{1}, \hat{P}_{2}, \hat{V}_{1}, \hat{V}_{2}\right\}$ is called a magnetic torus algebra without position operators.

Remark: The shift operators commute with the momenta, and therefore they characterize symmetry of the system.

Definition 2: quantization with position operators. Assume that there are self-adjoint operators $\hat{P}_{j}$ and unitary operators $\hat{U}_{j}(j=1,2)$ which satisfy the following relations

$$
\begin{aligned}
& {\left[\hat{P}_{1}, \hat{P}_{2}\right]=\frac{i m}{2 \pi} \hat{1},} \\
& \hat{U}_{1} \hat{U}_{2}=\hat{U}_{2} \hat{U}_{1}, \\
& {\left[\hat{P}_{j}, \hat{U}_{k}\right]=\delta_{j k} \hat{U}_{k}}
\end{aligned}
$$

The operators $\hat{U}_{j}$ are called position operators. The algebra $\mathcal{A}^{\prime}$ generated by $\left\{\hat{P}_{1}, \hat{P}_{2}, \hat{U}_{1}\right.$, $\left.\hat{U}_{2}\right\}$ is called a magnetic torus algebra with position operators.

Theorem 1: equivalence of the two quantizations. The algebra $\mathcal{A}^{\prime}$ is isomorphic to $\mathcal{A}$. Namely, there is a bijection $f: \mathcal{A}^{\prime} \rightarrow \mathcal{A}$ such that $f(\lambda \hat{A})=\lambda f(\hat{A}), f(\hat{A}+\hat{B})=f(\hat{A})+$ $f(\hat{B}), f(\hat{A} \hat{B})=f(\hat{A}) f(\hat{B})$ for any $\lambda \in \boldsymbol{C}$ and any $\hat{A}, \hat{B} \in \mathcal{A}^{\prime}$.

Remark: The above statement tells that any element $\hat{A} \in \mathcal{A}^{\prime}$ can be identified with $f(\hat{A}) \in \mathcal{A}$ but it does not say that $\hat{U}_{j}$ is identified with $\hat{V}_{j}$. Even if we write $f\left(\hat{U}_{j}\right)$ as $\hat{U}_{j}$, it does not cause confusion. Then they satisfy the relations

$$
\hat{U}_{j} \hat{V}_{k}=e^{2 \pi i \delta_{j k} / m} \hat{V}_{k} \hat{U}_{j} .
$$


Definition 3: momentum representation of the algebra. The space of measurable functions

$$
L^{2}(\boldsymbol{R}) \otimes \boldsymbol{C}^{|m|}:=\left\{\phi: \boldsymbol{R} \times\left.\boldsymbol{Z}_{m} \rightarrow \boldsymbol{C}\left|\int_{-\infty}^{\infty} d k \sum_{r=1}^{|m|}\right| \phi(k, r)\right|^{2}<\infty\right\}
$$

becomes a Hilbert space equipped with inner product

$$
\left\langle\phi_{1}, \phi_{2}\right\rangle:=\int_{-\infty}^{\infty} d k \sum_{r=1}^{|m|} \overline{\phi_{1}(k, r)} \phi_{2}(k, r), \quad \phi_{1}, \phi_{2} \in L^{2}(\boldsymbol{R}) \otimes \boldsymbol{C}^{|m|} .
$$

Here $r$ is an element of $\boldsymbol{Z}_{m}=\boldsymbol{Z} / m \boldsymbol{Z}$ and it is to be understood that $\phi(k, r+m)=\phi(k, r)$. Let the algebra $\mathcal{A}$ act on the Hilbert space $\mathcal{H}=L^{2}(\boldsymbol{R}) \otimes \boldsymbol{C}^{|m|}$ as

$$
\begin{aligned}
\hat{P}_{1} \phi(k, r) & =k \phi(k, r), \\
\hat{P}_{2} \phi(k, r) & =-\frac{i m}{2 \pi} \frac{\partial}{\partial k} \phi(k, r), \\
\hat{V}_{1} \phi(k, r) & =\phi(k, r-1), \\
\hat{V}_{2} \phi(k, r) & =e^{-2 \pi i r / m} \phi(k, r), \\
\hat{U}_{1} \phi(k, r) & =e^{2 \pi i r / m} \phi(k-1, r), \\
\hat{U}_{2} \phi(k, r) & =e^{2 \pi i k / m} \phi(k, r-1) .
\end{aligned}
$$

This is called a momentum representation.

Theorem 2: uniqueness of quantization. The momentum representation is an irreducible representation of the magnetic torus algebra $\mathcal{A}$. Moreover, any irreducible representation of $\mathcal{A}$ is unitarily equivalent to the momentum representation.

Definition 4: position representation of the algebra. Let $L_{m}^{2}\left(T^{2}\right)$ denote the space of measurable functions $\psi: \boldsymbol{R}^{2} \rightarrow \boldsymbol{C}$ such that

$$
\begin{aligned}
& \psi\left(q_{1}+2 \pi, q_{2}\right)=e^{i m q_{2}} \psi\left(q_{1}, q_{2}\right), \\
& \psi\left(q_{1}, q_{2}+2 \pi\right)=\psi\left(q_{1}, q_{2}\right), \\
& \int_{0}^{2 \pi} d q_{1} \int_{0}^{2 \pi} d q_{2}\left|\psi\left(q_{1}, q_{2}\right)\right|^{2}<\infty .
\end{aligned}
$$

The space $L_{m}^{2}\left(T^{2}\right)$ becomes a Hilbert space equipped with inner product

$$
\left\langle\psi_{1}, \psi_{2}\right\rangle:=\int_{0}^{2 \pi} d q_{1} \int_{0}^{2 \pi} d q_{2} \overline{\psi_{1}\left(q_{1}, q_{2}\right)} \psi_{2}\left(q_{1}, q_{2}\right), \quad \psi_{1}, \psi_{2} \in L_{m}^{2}\left(T^{2}\right) .
$$

Let the algebra $\mathcal{A}$ act on the Hilbert space $\tilde{\mathcal{H}}=L_{m}^{2}\left(T^{2}\right)$ as

$$
\begin{aligned}
& \hat{P}_{1} \psi\left(q_{1}, q_{2}\right)=-i \frac{\partial}{\partial q_{1}} \psi\left(q_{1}, q_{2}\right), \\
& \hat{P}_{2} \psi\left(q_{1}, q_{2}\right)=\left(-i \frac{\partial}{\partial q_{2}}-\frac{m}{2 \pi} q_{1}\right) \psi\left(q_{1}, q_{2}\right),
\end{aligned}
$$




$$
\begin{aligned}
& \hat{V}_{1} \psi\left(q_{1}, q_{2}\right)=e^{i q_{2}} \psi\left(q_{1}-\frac{2 \pi}{m}, q_{2}\right), \\
& \hat{V}_{2} \psi\left(q_{1}, q_{2}\right)=\psi\left(q_{1}, q_{2}-\frac{2 \pi}{m}\right), \\
& \hat{U}_{1} \psi\left(q_{1}, q_{2}\right)=e^{i q_{1}} \psi\left(q_{1}, q_{2}\right), \\
& \hat{U}_{2} \psi\left(q_{1}, q_{2}\right)=e^{i q_{2}} \psi\left(q_{1}, q_{2}\right) .
\end{aligned}
$$

This is called a position representation.

Remark: The wave function $\psi$ that satisfies the boundary condition (2.17) and (2.18) is essentially defined in the torus. Owing to (2.25) and (2.26), it is reasonable to call $\hat{U}_{j}$ position operators in the torus. In (2.21) and (2.22) the momentum operators are represented by the covariant derivative associated with a uniform magnetic field

$$
B=-i\left[\hat{P}_{1}, \hat{P}_{2}\right]=\frac{m}{2 \pi}
$$

The magnetic flux $\iint B d q_{1} d q_{2}=2 \pi m$ is quantized as a monopole in a sphere. Thus, it is natural to call the algebra $\mathcal{A}$ the magnetic torus algebra. Owing to (2.23) and (2.24), it is also reasonable to call $\hat{V}_{j}$ shift operators. They translate the wave function by a finite distance and they commute with the momentum operators. Therefore, the shift operators characterize symmetry of quantum mechanics in the torus as noted in the previous works $[8,9]$.

Theorem 3: The position representation is an irreducible representation of the magnetic torus algebra.

Remark: As a corollary of theorem 2, the position representation is also unitarily equivalent to the momentum representation. In this sense, quantum mechanics in the torus with the magnetic field is completely characterized by the algebra without position operators.

\section{Proof of theorem 1}

Assume that the operators $\left\{\hat{P}_{1}, \hat{P}_{2}, \hat{V}_{1}, \hat{V}_{2}\right\}$ satisfy the defining relations (2.1)-(2.4) of the algebra $\mathcal{A}$. If we put

$$
\hat{U}_{1}=e^{-2 \pi i \hat{P}_{2} / m} \cdot \hat{V}_{2}^{\dagger}, \quad \hat{U}_{2}=e^{2 \pi i \hat{P}_{1} / m} \cdot \hat{V}_{1},
$$

it is not difficult to verify that $\left\{\hat{P}_{1}, \hat{P}_{2}, \hat{U}_{1}, \hat{U}_{2}\right\}$ satisfy the defining relations (2.5)-(2.7) of the algebra $\mathcal{A}^{\prime}$. Thus we can say that $\mathcal{A}^{\prime} \subset \mathcal{A}$.

Oppositely, by accepting the relations of the algebra $\mathcal{A}^{\prime}$ and by putting

$$
\hat{V}_{1}=e^{-2 \pi i \hat{P}_{1} / m} \cdot \hat{U}_{2}, \quad \hat{V}_{2}=\hat{U}_{1}^{\dagger} \cdot e^{-2 \pi i \hat{P}_{2} / m},
$$


we can show that they satisfy the defining relations of the algebra $\mathcal{A}$. Thus we can say that $\mathcal{A} \subset \mathcal{A}^{\prime}$. Therefore, we conclude that $\mathcal{A}=\mathcal{A}^{\prime}$. The identity map id $: \mathcal{A}^{\prime} \rightarrow \mathcal{A}$ is actually taken to be the isomorphism $f: \mathcal{A}^{\prime} \rightarrow \mathcal{A}$. The relations (2.8) are also easily derived from (2.1)-(2.4) and (3.1).

\section{Proof of theorem 2}

It is easy to verify that the equations (2.11)-(2.16) define a representation of $\mathcal{A}$. Its irreducibility and uniqueness are proved below.

Suppose that we have any irreducible representation space of $\mathcal{A}$. The algebra $\mathcal{A}$ is generated by $\left\{\hat{P}_{1}, \hat{P}_{2}, \hat{V}_{1}, \hat{V}_{2}\right\}$. Two subalgebras generated by $\left\{\hat{P}_{1}, \hat{P}_{2}\right\}$ and by $\left\{\hat{V}_{1}, \hat{V}_{2}\right\}$ respectively are mutually commutative. Thus, it is enough for the proof to consider $\left\{\hat{P}_{1}, \hat{P}_{2}\right\}$ and $\left\{\hat{V}_{1}, \hat{V}_{2}\right\}$ separately. The representation of the whole algebra is given by a tensor product of representations of each subalgebra.

First, let us concentrate to the subalgebra generated by $\left\{\hat{P}_{1}, \hat{P}_{2}\right\}$. The defining relation (2.1) is isomorphic to the canonical commutation relation (CCR). It is well known 1 that any irreducible representation of the CCR is unitarily equivalent to the Schrödinger representation (2.11) and (2.12) over $L^{2}(\boldsymbol{R})$.

Second, let us turn to the subalgebra $\mathcal{B}$ generated by $\left\{\hat{V}_{1}, \hat{V}_{2}\right\}$. Take an orthonormal basis $\{|r\rangle|r=1,2, \cdots| m \mid$,$\} of \boldsymbol{C}^{|m|}$. If we let $\left\{\hat{V}_{1}, \hat{V}_{2}\right\}$ act on them as

$$
\hat{V}_{1}|r\rangle=|r+1\rangle, \quad \hat{V}_{2}|r\rangle=e^{-2 \pi i r / m}|r\rangle,
$$

these actions define a representation of $\mathcal{B}$ over $\boldsymbol{C}^{|m|}$. Here it is assumed that $|r+m\rangle=|r\rangle$.

We will show that the representation $\left(\mathcal{B}, C^{|m|}\right)$ is irreducible. Suppose that there exists an operator $\hat{T}$ which commutes with any element of $\mathcal{B}$. By taking matrix elements of $\left[\hat{T}, \hat{V}_{2}\right]=0$, we get

$$
e^{-2 \pi i r^{\prime} / m}\left\langle r|\hat{T}| r^{\prime}\right\rangle-e^{-2 \pi i r / m}\left\langle r|\hat{T}| r^{\prime}\right\rangle=\left(e^{-2 \pi i r^{\prime} / m}-e^{-2 \pi i r / m}\right)\left\langle r|\hat{T}| r^{\prime}\right\rangle=0 .
$$

Hence, $\left\langle r|\hat{T}| r^{\prime}\right\rangle=0$ when $r \neq r^{\prime}(\bmod m)$. On the other hand, by taking matrix elements of $\left[\hat{T}, \hat{V}_{1}\right]=0$, we get

$$
\left\langle r|\hat{T}| r^{\prime}+1\right\rangle-\left\langle r-1|\hat{T}| r^{\prime}\right\rangle=0
$$

which is equivalent to

$$
\left\langle r+1|\hat{T}| r^{\prime}+1\right\rangle=\left\langle r|\hat{T}| r^{\prime}\right\rangle .
$$

Therefore, the matrix $\left\langle r|\hat{T}| r^{\prime}\right\rangle$ is diagonal and their diagonal elements are equal. In other words, the operator $\hat{T}$ is a scalar. Then the Schur lemma implies that the representation $\left(\mathcal{B}, \boldsymbol{C}^{|m|}\right)$ is irreducible.

Next we will show that any other irreducible representation of $\mathcal{B}$ is unitarily equivalent to $\left(\mathcal{B}, \boldsymbol{C}^{|m|}\right)$. Suppose that a Hilbert space $\mathcal{E}$ provides an irreducible representation of $\mathcal{B}$. 
Then we define operators

$$
\hat{Q}_{r}:=\frac{1}{|m|} \sum_{q=1}^{|m|}\left(e^{2 \pi i r / m} \hat{V}_{2}\right)^{q}
$$

that act on $\mathcal{E}$. The set of operators $\left\{\hat{Q}_{1}, \cdots, \hat{Q}_{|m|}\right\}$ provides resolution of the identity. Namely, they satisfy

$$
\hat{Q}_{r} \hat{Q}_{r^{\prime}}=\delta_{r r^{\prime}} \hat{Q}_{r}, \quad\left(\hat{Q}_{r}\right)^{\dagger}=\hat{Q}_{r}, \quad \sum_{r=1}^{|m|} \hat{Q}_{r}=\hat{1} .
$$

These properties are checked by direct calculations: the first one is verified as

$$
\begin{aligned}
\hat{Q}_{r} \hat{Q}_{r^{\prime}} & =\frac{1}{|m|^{2}} \sum_{q, q^{\prime}=1}^{|m|}\left(e^{2 \pi i r / m} \hat{V}_{2}\right)^{q}\left(e^{2 \pi i r^{\prime} / m} \hat{V}_{2}\right)^{q^{\prime}} \\
& =\frac{1}{|m|^{2}} \sum_{q=1}^{|m|} e^{2 \pi i\left(r-r^{\prime}\right) q / m} \sum_{l=1}^{|m|} e^{2 \pi i r^{\prime} l / m}\left(\hat{V}_{2}\right)^{l} \\
& =\frac{1}{|m|^{2}}|m| \delta_{r r^{\prime}} \sum_{l=1}^{|m|}\left(e^{2 \pi i r^{\prime} / m} \hat{V}_{2}\right)^{l} \\
& =\delta_{r r^{\prime}} \hat{Q}_{r^{\prime}} .
\end{aligned}
$$

Here we put $q^{\prime}=l-q$. The second one is

$$
\left(\hat{Q}_{r}\right)^{\dagger}=\frac{1}{|m|} \sum_{q=1}^{|m|}\left(e^{2 \pi i r / m} \hat{V}_{2}\right)^{-q}=\hat{Q}_{r}
$$

The third one is

$$
\sum_{r=1}^{|m|} \hat{Q}_{r}=\frac{1}{|m|} \sum_{q=1}^{|m|}\left(\hat{V}_{2}\right)^{q} \sum_{r=1}^{|m|} e^{2 \pi i q r / m}=\sum_{q=1}^{|m|}\left(\hat{V}_{2}\right)^{q} \delta_{q 0}=\hat{1} .
$$

It is also easy to see

$$
\hat{V}_{2} \hat{Q}_{r}=e^{-2 \pi i r / m} \hat{Q}_{r}
$$

Hence the image of the projection operator $\hat{Q}_{r}$ is an eigenspace of $\hat{V}_{2}$ associated with the eigenvalue $e^{-2 \pi i r / m}$. Moreover, it is also easy to see

$$
\hat{V}_{1} \hat{Q}_{r} \hat{V}_{1}^{\dagger}=\hat{Q}_{r+1}
$$

which implies that all traces of $\hat{Q}_{r}(r=1, \cdots,|m|)$ are equal and hence the images of the projection operators $\hat{Q}_{r}$ have the equal dimensions $d=\hat{Q}_{r}(r=1, \cdots,|m|)$. Therefore, the representation $(\mathcal{B}, \mathcal{E})$ can be decomposed into $d$ copies of $\left(\mathcal{B}, C^{|m|}\right)$. If $(\mathcal{B}, \mathcal{E})$ is irreducible, $d=1$ and it is equivalent to $\left(\mathcal{B}, \boldsymbol{C}^{|m|}\right)$.

Combining above discussions, we conclude that the Hilbert space $L^{2}(\boldsymbol{R}) \otimes \boldsymbol{C}^{|m|}$ provides an irreducible representation of the algebra $\mathcal{A}$ and that any other irreducible representation of $\mathcal{A}$ is equivalent to it. 


\section{$5 \quad$ Proof of theorem 3}

Our plan of the proof is as follows: First, we will construct another irreducible representation $l^{2}(\boldsymbol{C}) \otimes \boldsymbol{C}^{|m|}$ of the algebra $\mathcal{A}$. Second, we will show that the momentum representation $L^{2}(\boldsymbol{R}) \otimes \boldsymbol{C}^{|m|}$ is unitarily equivalent to $l^{2}(\boldsymbol{C}) \otimes \boldsymbol{C}^{|m|}$. Third, we will show that the position representation $L_{m}^{2}\left(T^{2}\right)$ is unitarily equivalent to $l^{2}(\boldsymbol{C}) \otimes \boldsymbol{C}^{|m|}$. Combining them, we will complete the proof.

The first step: If we put

$$
\hat{a}:=\sqrt{\frac{\pi}{|m|}}\left(\hat{P}_{1}+i \frac{m}{|m|} \hat{P}_{2}\right), \quad \hat{a}^{\dagger}:=\sqrt{\frac{\pi}{|m|}}\left(\hat{P}_{1}-i \frac{m}{|m|} \hat{P}_{2}\right),
$$

they satisfy $\left[\hat{a}, \hat{a}^{\dagger}\right]=\hat{1}$. Let $\{|n, r\rangle|n=0,1,2, \cdots ; r=1,2, \cdots| m \mid$,$\} denote a complete$ orthonormal set of the Hilbert space $l^{2}(\boldsymbol{C}) \otimes \boldsymbol{C}^{|m|}$. The generators of the algebra $\mathcal{A}$ act as

$$
\begin{aligned}
& \hat{a}|n, r\rangle=\sqrt{n}|n-1, r\rangle, \quad \hat{a}^{\dagger}|n, r\rangle=\sqrt{n+1}|n+1, r\rangle, \\
& \hat{V}_{1}|n, r\rangle=|n, r+1\rangle, \quad \hat{V}_{2}|n, r\rangle=e^{-2 \pi i r / m}|n, r\rangle .
\end{aligned}
$$

It is well known that $l^{2}(\boldsymbol{C})$ provides an irreducible representation of the CCR. Let us call this representation an oscillator representation.

The second step: Putting $\phi_{n, r}\left(k, r^{\prime}\right)=\phi_{n}(k) \delta_{r r^{\prime}}=\left\langle k, r^{\prime} \mid n, r\right\rangle$ and combining (2.11), (2.12) with (5.2), we get the set of equations

$$
\begin{aligned}
& \sqrt{\frac{\pi}{|m|}}\left(k+\frac{|m|}{2 \pi} \frac{\partial}{\partial k}\right) \phi_{n}(k)=\sqrt{n} \phi_{n-1}(k), \\
& \sqrt{\frac{\pi}{|m|}}\left(k-\frac{|m|}{2 \pi} \frac{\partial}{\partial k}\right) \phi_{n}(k)=\sqrt{n+1} \phi_{n+1}(k) .
\end{aligned}
$$

The normalization condition $\int_{-\infty}^{\infty} d k\left|\phi_{n}(k)\right|^{2}=1$ is also imposed on them. The solution is uniquely given as

$$
\phi_{n}(k)=\frac{1}{\sqrt{2^{n} n !}}\left(\frac{2}{|m|}\right)^{\frac{1}{4}} e^{-\pi k^{2} /|m|} H_{n}\left(\sqrt{\frac{2 \pi}{|m|}} k\right)
$$

where $H_{n}(\xi)=(-1)^{n} e^{\xi^{2}} \frac{d^{n}}{d \xi^{n}} e^{-\xi^{2}}$ is the $n$-th Hermite polynomial. The set of functions $\left\{\phi_{n, r}|n=0,1,2, \cdots ; r=1,2, \cdots| m \mid,\right\}$ constitutes a complete orthonormal set of $L^{2}(\boldsymbol{R}) \otimes$ $\boldsymbol{C}^{|m|}$. Then the linear map

$$
\Gamma_{1}: l^{2}(\boldsymbol{C}) \otimes \boldsymbol{C}^{|m|} \rightarrow L^{2}(\boldsymbol{R}) \otimes \boldsymbol{C}^{|m|}, \quad \sum_{n=0}^{\infty} \sum_{r=1}^{|m|} c_{n, r}|n, r\rangle \mapsto \sum_{n=0}^{\infty} \sum_{r=1}^{|m|} c_{n, r} \phi_{n, r}\left(k, r^{\prime}\right)
$$

becomes a unitary transformation that bridges the oscillator representation and the momentum representation. 
The third step: Putting $\psi_{n, r}\left(q_{1}, q_{2}\right)=\left\langle q_{1}, q_{2} \mid n, r\right\rangle$ and combining (2.21)-(2.24) with (5.2), (5.3), we get the set of equations

$$
\begin{aligned}
& \sqrt{\frac{\pi}{|m|}}\left(-i \frac{\partial}{\partial q_{1}}+\frac{m}{|m|}\left(\frac{\partial}{\partial q_{2}}-i \frac{m}{2 \pi} q_{1}\right)\right) \psi_{n, r}\left(q_{1}, q_{2}\right)=\sqrt{n} \psi_{n-1, r}\left(q_{1}, q_{2}\right) \\
& \sqrt{\frac{\pi}{|m|}}\left(-i \frac{\partial}{\partial q_{1}}-\frac{m}{|m|}\left(\frac{\partial}{\partial q_{2}}-i \frac{m}{2 \pi} q_{1}\right)\right) \psi_{n, r}\left(q_{1}, q_{2}\right)=\sqrt{n+1} \psi_{n+1, r}\left(q_{1}, q_{2}\right), \\
& e^{i q_{2}} \psi_{n, r}\left(q_{1}-\frac{2 \pi}{m}, q_{2}\right)=\psi_{n, r+1}\left(q_{1}, q_{2}\right) \\
& \psi_{n, r}\left(q_{1}, q_{2}-\frac{2 \pi}{m}\right)=e^{-2 \pi i r / m} \psi_{n, r}\left(q_{1}, q_{2}\right) .
\end{aligned}
$$

The boundary condition (2.17), (2.18) and the normalization condition $\int_{0}^{2 \pi} d q_{1} d q_{2} \mid \psi_{n, r}\left(q_{1}\right.$, $\left.q_{2}\right)\left.\right|^{2}=1$ are imposed on them. By a tedious calculation 9 we get the unique solution

$$
\begin{aligned}
\psi_{n, r}\left(q_{1}, q_{2}\right)= & \frac{i^{n}}{\sqrt{2^{n} n !}}(2|m|)^{\frac{1}{4}} \sum_{l=-\infty}^{\infty} e^{i(m l+r) q_{2}} \\
& e^{-|m|\left\{q_{1}-2 \pi(m l+r) / m\right\}^{2} /(4 \pi)} H_{n}\left[\sqrt{\frac{|m|}{2 \pi}}\left\{q_{1}-2 \pi(m l+r) / m\right\}\right] .
\end{aligned}
$$

The set of functions $\left\{\psi_{n, r}|n=0,1,2, \cdots ; r=1,2, \cdots| m \mid,\right\}$ constitutes a complete orthonormal set of $L_{m}^{2}\left(T^{2}\right)$. Then the linear map

$$
\Gamma_{2}: l^{2}(\boldsymbol{C}) \otimes \boldsymbol{C}^{|m|} \rightarrow L_{m}^{2}\left(T^{2}\right), \quad \sum_{n=0}^{\infty} \sum_{r=1}^{|m|} c_{n, r}|n, r\rangle \mapsto \sum_{n=0}^{\infty} \sum_{r=1}^{|m|} c_{n, r} \psi_{n, r}\left(q_{1}, q_{2}\right)
$$

becomes a unitary transformation that bridges the oscillator representation and the position representation.

The final step: It is obvious that the combined map $\Gamma=\Gamma_{2} \circ \Gamma_{1}^{-1}$ is a unitary transformation which transforms the momentum representation to the position representation.

\section{Concluding remarks}

We leave some remarks in order. The unitary transformation $\Gamma: L^{2}(\boldsymbol{R}) \otimes \boldsymbol{C}^{|m|} \rightarrow L_{m}^{2}\left(T^{2}\right)$ is concretely given [9] as

$$
\psi\left(q_{1}, q_{2}\right)=\sum_{l=-\infty}^{\infty} \sum_{r=1}^{|m|} \int_{-\infty}^{\infty} d k e^{i k\left(q_{1}-2 \pi(m l+r) / m\right)+i(m l+r) q_{2}} \phi(k, r) .
$$

The transforming function

$$
\chi_{k, r}\left(q_{1}, q_{2}\right)=\left\langle q_{1}, q_{2} \mid k, r\right\rangle=\sum_{l=-\infty}^{\infty} e^{i k\left(q_{1}-2 \pi(m l+r) / m\right)+i(m l+r) q_{2}}
$$


is a formal solution of the equations

$$
\begin{aligned}
& -i \frac{\partial}{\partial q_{1}} \chi_{k, r}\left(q_{1}, q_{2}\right)=k \chi_{k, r}\left(q_{1}, q_{2}\right), \\
& \left(-i \frac{\partial}{\partial q_{2}}-\frac{m}{2 \pi} q_{1}\right) \chi_{k, r}\left(q_{1}, q_{2}\right)=\frac{i m}{2 \pi} \frac{\partial}{\partial k} \chi_{k, r}\left(q_{1}, q_{2}\right), \\
& e^{i q_{2}} \chi_{k, r}\left(q_{1}-\frac{2 \pi}{m}, q_{2}\right)=\chi_{k,(r+1)}\left(q_{1}, q_{2}\right), \\
& \chi_{k, r}\left(q_{1}, q_{2}-\frac{2 \pi}{m}\right)=e^{-2 \pi i r / m} \chi_{k, r}\left(q_{1}, q_{2}\right), \\
& \chi_{k,(r+m)}\left(q_{1}, q_{2}\right)=\chi_{k, r}\left(q_{1}, q_{2}\right) .
\end{aligned}
$$

However, the infinite sum with respect to $l$ in (6.2) does not converge and hence $\chi_{k, r}$ is called a 'formal' solution. It is a natural consequence of the fact that the operator $\hat{P}_{1}$ has a continuous spectrum $-\infty<k<\infty$ and the generally-known fact that there is no normalizable eigenfunction for a continuous spectrum. It is to be noted that the spectrum of the momentum is not discrete although the torus is a compact space.

We shall mention another representation of the algebra $\mathcal{A}$. If we replace (2.21) $-(2.26)$ by

$$
\begin{aligned}
\rho_{\alpha}\left(\hat{P}_{1}\right) \psi\left(q_{1}, q_{2}\right) & =\left(-i \frac{\partial}{\partial q_{1}}-\alpha_{1}\right) \psi\left(q_{1}, q_{2}\right) \\
\rho_{\alpha}\left(\hat{P}_{2}\right) \psi\left(q_{1}, q_{2}\right) & =\left(-i \frac{\partial}{\partial q_{2}}-\frac{m}{2 \pi} q_{1}-\alpha_{2}\right) \psi\left(q_{1}, q_{2}\right), \\
\rho_{\alpha}\left(\hat{U}_{1}\right) \psi\left(q_{1}, q_{2}\right) & =e^{i\left(q_{1}+2 \pi \alpha_{2} / m\right)} \psi\left(q_{1}, q_{2}\right), \\
\rho_{\alpha}\left(\hat{U}_{2}\right) \psi\left(q_{1}, q_{2}\right) & =e^{i\left(q_{2}-2 \pi \alpha_{1} / m\right)} \psi\left(q_{1}, q_{2}\right),
\end{aligned}
$$

with leaving $\rho_{\alpha}\left(\hat{V}_{j}\right)=\hat{V}_{j}$, we get another irreducible representation. Here $\left(\alpha_{1}, \alpha_{2}\right) \in \boldsymbol{R}^{2}$ are arbitrary parameters, which cause the Aharonov-Bohm effect. However, it is again unitarily equivalent to (2.21) $-(2.26)$ as seen below. If we introduce

$$
\hat{S}_{\alpha} \psi\left(q_{1}, q_{2}\right)=e^{i \alpha_{1} q_{1}} \psi\left(q_{1}+\frac{2 \pi}{m} \alpha_{2}, q_{2}-\frac{2 \pi}{m} \alpha_{1}\right)
$$

then $\hat{S}_{\alpha}$ is a unitary operator acting on $L_{m}^{2}\left(T^{2}\right)$ and it satisfies

$$
\hat{S}_{\alpha} \hat{P}_{j} \hat{S}_{\alpha}^{-1}=\rho_{\alpha}\left(\hat{P}_{j}\right), \quad \hat{S}_{\alpha} \hat{V}_{j} \hat{S}_{\alpha}^{-1}=\rho_{\alpha}\left(\hat{V}_{j}\right), \quad \hat{S}_{\alpha} \hat{U}_{j} \hat{S}_{\alpha}^{-1}=\rho_{\alpha}\left(\hat{U}_{j}\right)
$$

Hence we can conclude that the representation (6.8)-(6.11) is unitarily equivalent to to the original one defined by (2.21)-(2.26). It is to be noted that the operator (6.12) makes sense only when $m$ is nonzero. If $m=0$ and $\alpha_{j}$ is not an integer, there is no unitary operator which transforms $\hat{P}_{j}$ to $\rho_{\alpha}\left(\hat{P}_{j}\right)$.

In our formulation, the Hamiltonian (1.3), which was not well defined in $T^{2}$, is correctly replaced by

$$
\hat{H}=\frac{1}{2}\left(\hat{P}_{1}\right)^{2}+\frac{1}{2}\left(\hat{P}_{2}\right)^{2} .
$$


Its spectrum yields the Landau levels and the eigenfunctions have been written down as (5.6) and (5.12). We close our discussion by mentioning that the magnetic torus algebra, which is defined by the relations (2.1)-(2.8), is equivalent to the so-called noncommutative torus algebra[10]. However, our formulation has clear definitions and an reasonable interpretation. Moreover, it reveals the new fact that quantization in a topologically nontrivial space is possible without resorting to position operators. These points are advantages of our formulation.

Let us put a final comment; in our formulation the gauge field is assumed to be a fixed background. It is desirable to extend the formulation to field theory for more realistic application. For this point, Ho and Hosotani 11 have examined in detail the translation symmetry of the Chern-Simons gauge system in a torus.

\section{Acknowledgments}

I have learned much about quantum mechanics in manifolds from collaborations with I. Tsutsui and M. Sakamoto. I wish to thank them. M. Takeori called my attention to the uniqueness problem of quantization and my interest in the problem grew from discussions with him. M. Nakahara invited me to write an introductory article on topology and quantum mechanics. When I was preparing the article and re-examining my previous work on quantum mechanics in the torus, I reached the conclusion that I present here. I thank Takeori and Nakahara for making opportunity of this work. I am grateful to people who have been encouraging me at various aspects. Discussions during the workshop YITP-W-03-07 on "QFT2003" at the Yukawa Institute for Theoretical Physics were also useful to complete this work.

\section{References}

[1] J. von Neumann, Ann, Math. 104 (1931) 570.

[2] H. Reeh, "A remark concerning canonical commutation relations", J. Math. Phys. 29 (1988) 1535.

[3] A. Arai, "Momentum operators with gauge potentials, local quantization of magnetic flux, and representation of canonical commutation relations", J. Math. Phys. 33 (1992) 3374.

[4] G. W. Mackey, Induced representations of groups and quantum mechanics, (Benjamin, New York 1968).

[5] C. J. Isham, "Topological and global aspects of quantum theory", in Relativity, Groups and Topology II, B. S. DeWitt and R. Stora, Eds (North-Holland, Amsterdam, 1984) 1059. 
[6] Y. Ohnuki, S. Kitakado, "Fundamental algebra for quantum mechanics on $S^{D}$ and gauge potentials", J. Math. Phys. 34 (1993) 2827.

[7] S. Tanimura, "Gauge field, parity and uncertainty relation of quantum mechanics on $S^{1}$ ”, Prog. Theor. Phys. 90 (1993) 271; arXiv hep-th/9306098.

[8] S. Tanimura, "Magnetic translation groups in $n$-dimensional torus and their representations", J. Math. Phys. 43, 5926 (2002); arXiv hep-th/0205053.

[9] M. Sakamoto, S. Tanimura, "An extension of Fourier analysis for the $n$-torus in the magnetic field and its application to spectral analysis of the magnetic Laplacian", J. Math. Phys. (in press); arXiv hep-th/0306006.

[10] A. Connes, M. R. Douglas, A. Schwarz, "Noncommutative Geometry and Matrix Theory: Compactification on Tori", JHEP 9802, 003 (1998); arXiv hep-th/9711162.

[11] C-L. Ho, Y. Hosotani, "Operator algebra in Chern-Simons theory on a torus", Phys. Rev. Lett. 701360 (1993); arXiv hep-th/9210103. 